\title{
Follow-Up Period
}

National Cancer Institute

\section{Source}

National Cancer Institute. Follow-Up Period. NCI Thesaurus. Code C156841.

The period of time determined to be follow-up. 